\title{
CHARACTERIZATION OF DUAL-AXIS PIEZORESISTIVE MEMS FORCE SENSORS
}

\author{
G.C. Hill, D.R. Soto, J.E. Oliver, and T.W. Kenny \\ Stanford University, Stanford, California, USA
}

\begin{abstract}
Dual-axis piezoresistive MEMS force sensors were rigorously characterized to improve their calibration. Displacement sensitivity was measured directly as in [1], while spring constants were calculated using resonant frequency measurements and finite element modeling (FEM) [1, 2]. Similar devices have been described in the literature [3,4], but this is the first report of a robust calibration process that corrects for the expected variability of critical parameters.
\end{abstract}

\section{INTRODUCTION}

The stiffness of MEMS cantilevers is difficult to measure directly. Some have fabricated and calibrated reference springs for this purpose[5], a method limited by the reference calibration and the ability to control the orientation and position of interaction. The use of analytical formulas for stiffness predictions is limited by the applicability of the formula and the accuracy of material and geometry parameters.

These dual-axis sensors have distinct regions of lateral and normal compliance (Figure 1). The tip half is $1 \mu \mathrm{m}$-tall and bends

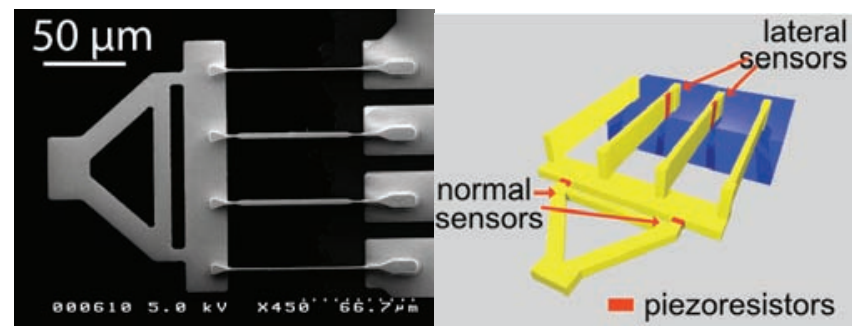

Figure 1: SEM of cantilever (left) and schematic (right) showing piezoresistive implants that transduce forces applied at the tip. Sensors are located in regions of high stress under deflection (see Figure 2). In the SEM, "normal" forces are out of the page, while "lateral" forces are vertical on the page.
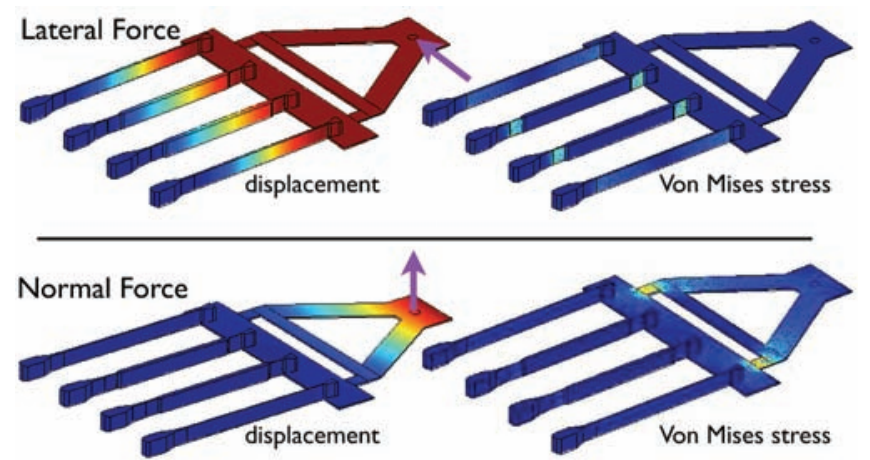

Figure 2: Finite element model (FEM) of a cantilever with force applied laterally (top) and normally (bottom), where color shows displacement (left) and von Mises stress (right). Lateral compliance is achieved primarily in the long, thin "ribs", with stress concentrated at the location of the lateral sensors, and normal compliance is primarily in the triangular tip, with stress concentrated at the normal sensors. in response to normally applied forces, while resisting lateral forces (Figure 2). Four tall $(10 \mu \mathrm{m})$, skinny $(0.75-1.5 \mu \mathrm{m})$, long $(80-200 \mu \mathrm{m})$ ribs connect the triangle tip to the base. These ribs comply with lateral forces but are stiff to normal forces. Piezoresistors are strategically implanted in high-stress regions, and are read by a Wheatstone-bridge signal conditioning circuit.

\section{RESULTS}

\section{Displacement sensitivity}

The voltage output from the signal conditioning circuit is linearly related to tip deflection by the displacement sensitivity parameter. Displacement sensitivities of $\sim 0.1-1 \mathrm{~V} / \mu \mathrm{m}$ were measured using a computer-controlled piezoelectric stage to move the cantilever into a fixed bristle (Figure 3). These sensitivities were highly reproducible, with less than $1 \%$ variation in successive measurements at the same point (Figure 4). Sensitivities varied by $5 \%$ (normal direction) and $10 \%$ (lateral direction) with repositioning. In particular, applying normal forces offset from the tip center did not affect displacement sensitivity (Figure 5). Sensitivity measurements made over several months do not show drift greater than the repositioning variability. Thus, once measured, the displacement sensitivity may be used with confidence in later experiments.

\section{Force constants}

A 3-D FEM (COMSOL 3.3a) was constructed for each cantilever and used to predict its lateral and normal stiffnesses and resonant frequencies. Input parameters included mask dimensions and bulk silicon material properties. The critical dimension in each analysis (rib thickness $t_{r i b}$ for lateral, cantilever thickness $t_{c a n t}$ for normal) was not well described by mask dimensions. Both these dimensions are set in a timed $9 \mu$ m-etch of the ribs pattern into the $10 \mu \mathrm{m}$-device layer of a silicon-on-insulator wafer. The remaining " $1 \mu \mathrm{m}$ " device layer is the nominal cantilever thickness; however, $5 \%$ etch rate variability across a wafer results in cantilever thicknesses from 0.55 to $1.45 \mu \mathrm{m}$. Similar variability was noted during fabrication, but cantilever thicknesses were difficult to measure precisely after processing. SEM measurements of rib thicknesses were taken and showed significant thinning from the mask dimensions $(\sim 50 \%)$, as well as significant variability in thickness along each rib.

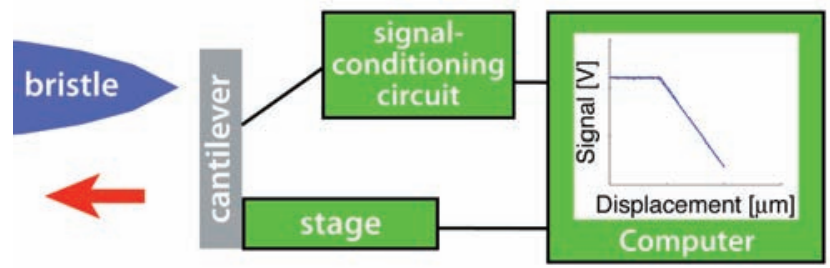

Figure 3: Experimental set-up for displacement sensitivity measurement. Cantilever is mounted on a computer-controlled piezoelectric stage and moved into a bristle. As the cantilever deflects, a Wheatstone bridge signal-conditioning circuit measures the change in piezoresistance. The displacement sensitivity is the slope of a linear fit to voltage output vs. displacement. 
Table 1: Fitting geometry parameters in a finite element model (FEM) to measured resonant frequencies. Lateral- and normal-mode resonant frequencies were measured for each cantilever using a laser-Doppler vibrometer. Model 1 uses average SEM measurements for rib thickness $\mathrm{t}_{\mathrm{rib}}$ and the nominal cantilever thickness $\mathrm{t}_{\mathrm{cant}}=1 \mu \mathrm{m}$. Predicted frequencies are off by an average of $20 \%$ (normal-mode) and 90\% (lateral). Model 2 uses the optimized thicknesses for each cantilever (see Figure 6) such that modeled resonant frequencies match observation within 1-2\%. Frequencies are in $\mathrm{kHz}$ and dimensions in $\mu \mathrm{m}$. Stiffness parameters from Model 2 are to be used in future work.

\begin{tabular}{|c|c|c|c|c|c|c|c|c|c|c|}
\hline \multirow[t]{2}{*}{ Cantilever } & \multicolumn{2}{|c|}{ Measured } & \multicolumn{4}{|c|}{ Model 1} & \multicolumn{4}{|c|}{ Model 2} \\
\hline & $f_{\text {lat }}$ & $f_{\text {norm }}$ & $t_{r i b}$ & $t_{\text {cant }}$ & $f_{\text {lat }}$ & $f_{\text {norm }}$ & $t_{r i b}$ & $t_{\text {cant }}$ & $f_{\text {lat }}$ & $f_{\text {norm }}$ \\
\hline 1 & 122 & 204 & 1.44 & 1.00 & 198 & 185 & 1.01 & 1.11 & 122 & 204 \\
\hline 2 & 52 & 92 & 1.06 & 1.00 & 107 & 93 & 0.62 & 0.98 & 52 & 92 \\
\hline 3 & 20 & 59 & 0.97 & 1.00 & 55 & 68 & 0.46 & 0.87 & 20 & 59 \\
\hline 4 & 14 & 41 & 0.95 & 1.00 & 29 & 45 & 0.55 & 0.89 & 14 & 41 \\
\hline 5 & 63 & 72 & 0.87 & 1.00 & 83 & 92 & 0.67 & 0.76 & 63 & 72 \\
\hline 6 & 25 & 68 & 0.79 & 1.00 & 42 & 89 & 0.51 & 0.73 & 25 & 68 \\
\hline 7 & 24 & 50 & 0.79 & 1.00 & 41 & 67 & 0.49 & 0.72 & 24 & 50 \\
\hline 8 & 36 & 64 & 0.75 & 1.00 & 68 & 92 & 0.44 & 0.67 & 36 & 64 \\
\hline
\end{tabular}

Normal displacement sensitivity measurements: repeatability without repositioning

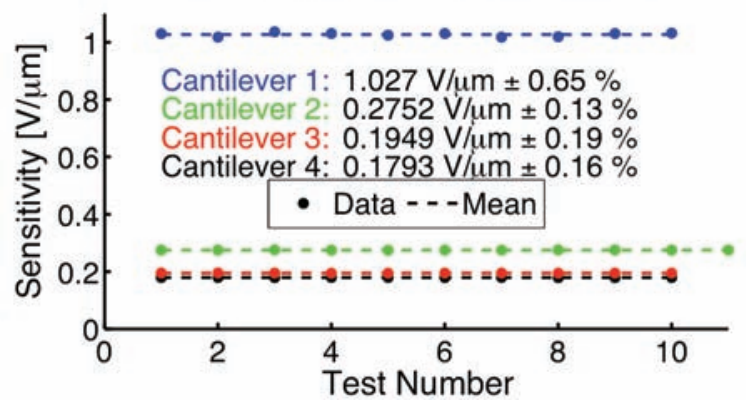

Figure 4: Displacement sensitivity measurements are highly reproducible (standard deviation $<1 \%$ ) if the cantilever is not repositioned between measurements. Ten measurements each of four cantilevers shown. Errors are standard deviations of values.

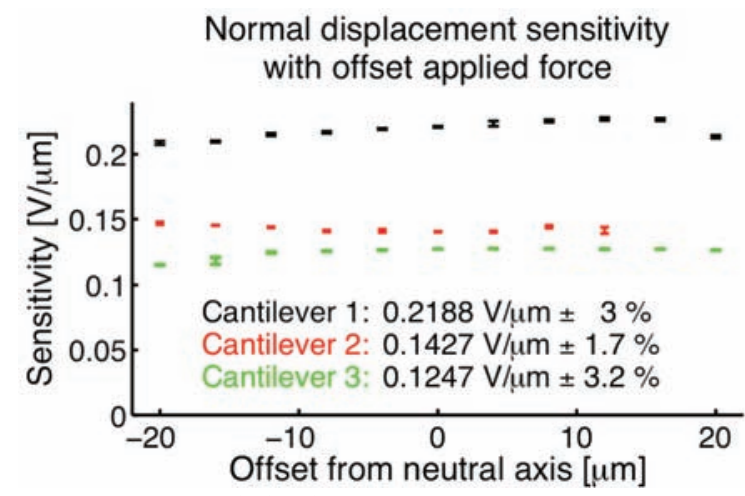

Figure 5: Normal displacement sensitivity measurements versus offset from neutral axis along cantilever tip. Averages shown with error bars. Sensitivity is only weakly related to offset.

Initial models used the measured rib thicknesses and nominal cantilever thickness (Table 1). The predicted lateral and normal resonant frequencies of each device were then compared to actual resonant frequencies measured with a laser-Doppler vibrometer. The predictions were off by $20 \%$ (normal-mode) and $90 \%$ (lateralmode), revealing modest inaccuracies in $t_{\text {cant }}$ and significant inaccuracies in $t_{r i b}$. This result, consistent with the observed variable rib thinning, suggests that the visually observed rib thicknesses were greater than the mechanically effective rib thicknesses. For each mode of each device, the critical parameter was adjusted until the resonant frequency predicted by the model matched observation (Figure 6).
Fitting cantilever thickness to normal-mode resonant frequency

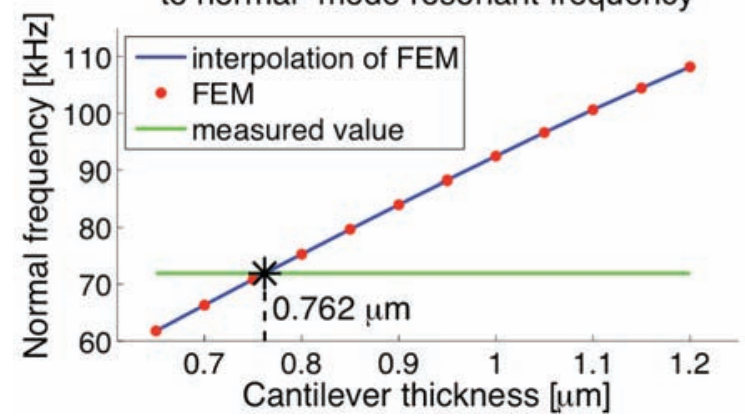

Figure 6: The cantilever thickness in an FEM is adjusted to match the observed normal-mode resonant frequency for a specific cantilever. This model fits at a cantilever thickness of $0.762 \mu \mathrm{m}$.

\section{CONCLUSIONS}

In conjunction with the measured displacement sensitivities, using stiffnesses predicted by resonant frequency-corrected models will improve the accuracy of two-component force measurements made with these sensors in future experiments.

\section{ACKNOWLEDGEMENTS}

The National Science Foundation partially supported this work under grants ECS-9731293 and EEC-0425914. A Kirby Stanford Graduate Fellowship and Stanford's Summer Undergraduate Research Institute partially supported G.H. and J.O.

\section{REFERENCES}

[1] T. Gotszalk, P. Grabiec, and I. W. Rangelow, "Calibration and examination of piezoresistive Wheatstone bridge cantilevers for scanning probe microscopy", Ultramicroscopy, 97, 385-9, (2003).

[2] J. L. Hazel and V. V. Tsukruk, "Friction force microscopy measurements: normal and torsional spring constants for Vshaped cantilevers", J. Tribology, 120, 814-19, (1998).

[3] K. Autumn, Y. A. Liang, S. T. Hsieh, W. Zesch, W. P. Chan, T. W. Kenny, R. Fearing, and R. J. Full, "Adhesive force of a single gecko foot-hair." Nature, 405, 681-5, (2000).

[4] B. W. Chui, T. W. Kenny, H. J. Mamin, B. D. Terris, and D. Rugar, "Independent detection of vertical and lateral forces with a sidewall-implanted dual-axis piezoresistive cantilever", Applied Physics Letters, 72, 1388-1390, (1998).

[5] P. J. Cumpson, P. Zhdan, and J. Hedley, "Calibration of AFM cantilever stiffness: a microfabricated array of reflective springs", Ultramicroscopy, 100, 241-51, (2004). 